\title{
An Experimental Study of the Phase Stability of VLF Signals
}

\author{
D. E. Hampton
}

\author{
Contribution From Ministry of Aviation, Royal Aircraft Establishment, Radio Department, S. Farnborough, \\ Hampshire, England
}

A large amount of experimental evidence is now available on both the field strength and phase of VLF waves which have traveled over a wide variety of paths. Much effort has been made to sift this information to reveal the unusual effects due to ionosphere disturbances of one sort or another. The bulk of the data, however, exhibits the remarkable stability of both the phase and amplitude of VLF signals from day to day, and it is these records which are examined in this paper. Since the phase of the signal is the characteristic which can be exploited most for practical application, particularly navigation systems, this paper is primarily concerned with the diurnal variation of phase.

It has been shown [Blackband, 1961] that for signals propagated over distances greater than about $4,000 \mathrm{~km}$, the difference in phase from conditions when the whole path is in daylight is linearly dependent on distance. This is in agreement with the idea of a single waveguide mode whose velocity depends on the height of ionosphere which assumes one constant value throughout the day and another throughout the night.

For conditions when the path is part light and part dark, a simple model is to assume a step in the ionosphere height at the points of dawn and dusk along the path and that the phase velocity is discontinuous across these points having a value $V_{1}$ in the light and a value $V_{2}$ in the dark (velocity in $\mathrm{km} / \mathrm{sec}$ ). Such a model is shown in this paper to give a remarkably good fit to the experimental data on the longer distance paths.

For such a model, the phase delay, $\tau \mu$ secs over a distance of $D \mathrm{~km}$ when a proportion " $p$ " of the path is in darkness can be shown to be given by

$$
\tau=\tau_{0}+p \Delta \tau
$$

where $\tau_{0}=\frac{D}{v_{1}}$ is the phase delay when all the path is in light. $\Delta \tau=D\left(\frac{1}{V_{2}}-\frac{1}{V_{1}}\right)$ is the change in delay when the whole path goes into darkness.

It is assumed that in (1) the only parameter depending on the time of day is " $p$ " can be calculated from the geometry of receiver position, transmitter position and the direction of the sun, the shape of the diurnal variation curve can be readily obtained.

The simplest results to interpret are those obtained from paths which do not cross the Arctic or Antarctic circle. At one time of the day, these paths are in light, while at another time they are in darkness so that the diurnal variation follows the well-known trapezoidal form. The depth of the trapezium is a measure of $\Delta \tau$ the total diurnal change.

The path from Rugby, England (GBR $16 \mathrm{kc} / \mathrm{s}$ ), to Singapore is mainly over land and is about 11,000 $\mathrm{km}$. Values of $\Delta \tau$ for each day for one year show a seasonal trend which is almost concealed by a dayto-day random variation of about 10 percent of the mean value. Other records show a similar random variation. This could be an instrumental error due to the instability of either the transmitted frequency or the reference frequency used on the ground monitor for it corresponds approximately to a variation in the 24-hr mean frequency of 1 part in $10^{-10}$ per day.

Paths which cross the polar regions are somewhat more difficult to interpret since the path does not generally change from being in complete darkness to complete light within $24 \mathrm{hr}$. The transmission path of signals from NPM Hawaii received at Farnborough, England $(11,600 \mathrm{~km})$, passes within the Arctic Circle and the phase variation of these signals can only be interpreted by calculating the proportion of the path in darkness for the particular time of year at which the records are obtained. It has been found that (1) fits the experimental results best if the calculation of " $p$ " is made so that the positions of dawn and dusk along the path occur at an altitude of 


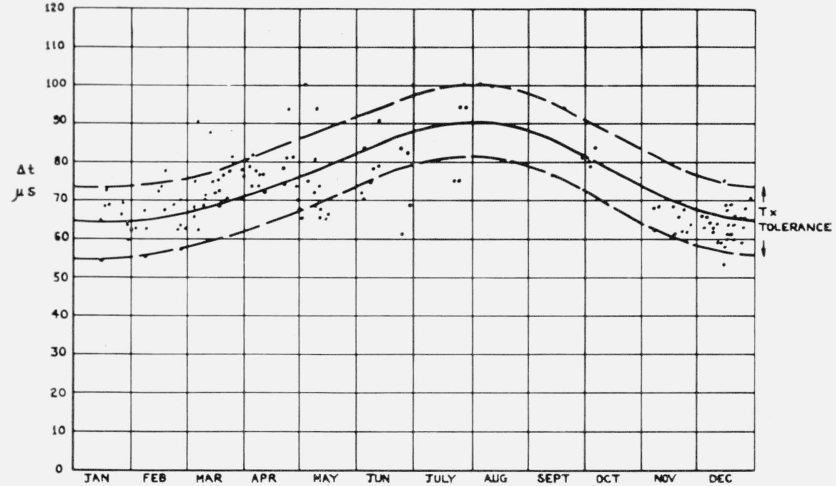

Figure 1. Diurnal phase changes of GBR received at Singapore, $10900 \mathrm{~km}$.

$60 \mathrm{~km} / \mathrm{s}$ above the ozonosphere. The calculated curves fit the experimental curves to within a few microseconds but the estimates of $\Delta \tau$ obtained exhibit the same day-to-day fluctuations as have been obtained on nonpolar paths.

Average values of $\Delta \tau$ over a week or so, when divided by the path length, gives estimates of $\left(\frac{1}{V_{1}}-\frac{1}{V_{2}}\right)$ which depends on frequency. These results are in general agreement with mode theory calculations [Wait, 1962].

\section{References}

Blackband, W. T. (Nov.-Dec. 1961), Effects of the ionosphere on VLF navigational aids, J. Res. 65D (Radio Prop.), No. $6,575-580$.

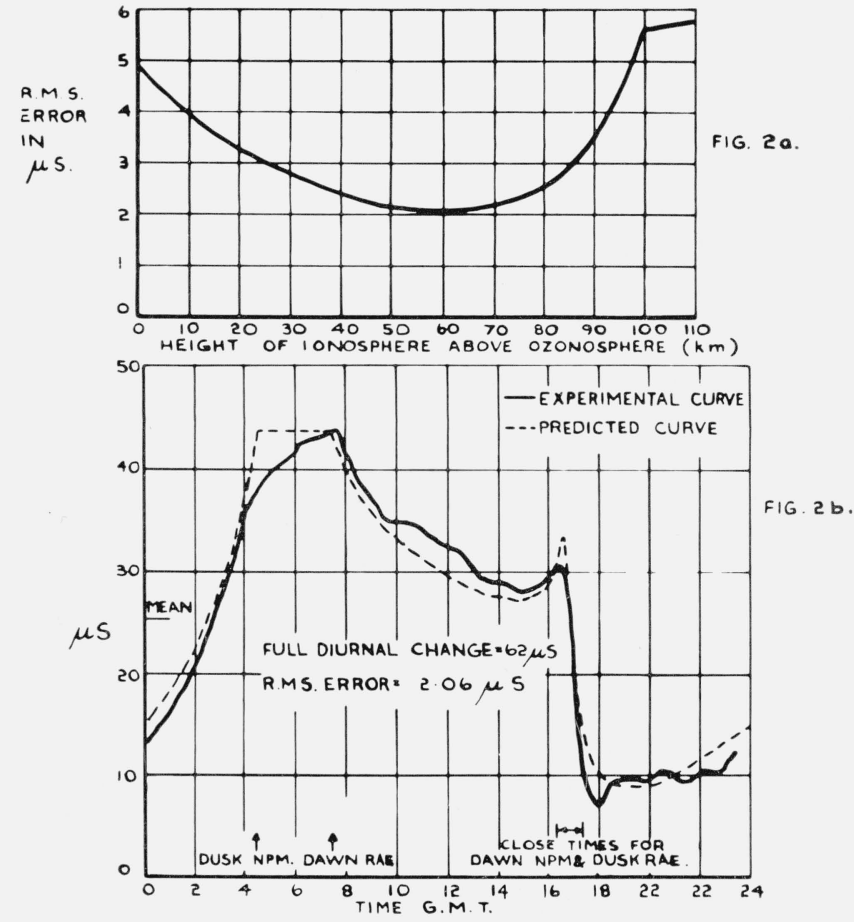

FIGURE 2. Analysis of mean curve for 22d to 31st December 1962 for path NPM Hawaii to Rae Farnborough.

Length of path $11600 \mathrm{~km}$.

Wait, J. R. (1962), Electromagnetic Waves in Stratified Media (Pergamon Press, Oxford).

(Paper 68D1-311) 